\title{
THE
}

\section{Transition Rates via Bethe Ansatz for the Spin-1/2 Heisenberg Chain}

\author{
Daniel Biegel \\ Michael Karbach \\ University of Rhode Island \\ Gerhard Müller \\ University of Rhode Island, gmuller@uri.edu
}

Follow this and additional works at: https://digitalcommons.uri.edu/phys_facpubs

Terms of Use

All rights reserved under copyright.

\section{Citation/Publisher Attribution}

Biegel, D., Karbach, M., \& Müller, G. (2002). Transition rates via Bethe ansatz for the spin-1/2 Heisenberg chain. Europhysics Letters, 59(6), 882-888. doi: 10.1209/epl/i2002-00125-0

Available at: http://dx.doi.org/10.1209/epl/i2002-00125-0

This Article is brought to you for free and open access by the Physics at DigitalCommons@URI. It has been accepted for inclusion in Physics Faculty Publications by an authorized administrator of DigitalCommons@URI. For more information, please contact digitalcommons-group@uri.edu. 
EPJ manuscript No.

(will be inserted by the editor)

\title{
Transition rates via Bethe ansatz for the spin- $\frac{1}{2}$ Heisenberg chain
}

\author{
Daniel Biegel ${ }^{1}$, Michael Karbach ${ }^{1}$, and Gerhard Müller ${ }^{2}$ \\ Bergische Universität Wuppertal, Fachbereich Physik, D-42097 Wuppertal, Germany, e-mail: michael@karbach.org \\ Department of Physics, University of Rhode Island, Kingston RI 02881-0817, USA, e-mail: gmuller@uri.edu
}

February 1, 2008

\begin{abstract}
We use the exact determinantal representation derived by Kitanine, Maillet, and Terras for matrix elements of local spin operators between Bethe wave functions of the one-dimensional $s=\frac{1}{2}$ Heisenberg model to calculate and numerically evaluate transition rates pertaining to dynamic spin structure factors. For real solutions $z_{1}, \ldots, z_{r}$ of the Bethe ansatz equations, the size of the determinants is of order $r \times r$. We present applications to the zero-temperature spin fluctuations parallel and perpendicular to an external magnetic field.
\end{abstract}

\section{Introduction}

The one-dimensional (1D) $s=\frac{1}{2}$ Heisenberg model was solved, in principle, by Bethe some 70 years ago using an ad-hoc trial wave function, now famously known as the Bethe ansatz [1]. Decades went by before the power and scope of this method of exact analysis became widely known for applications to spectrum and thermodynamics of a select class of completely integrable model systems [2].

Until recently, the most vexing exception to immense progress in the further development of the Bethe ansatz has been the absence of a practical method to use the exactly known and readily available Bethe wave functions for the explicit calculation of transition matrix elements. The knowledge of such transition rates is of paramount importance for an understanding of dynamic correlation functions in relation to the underlying quasiparticles and for the interpretation of experimental probes of quantum fluctuations in quasi-1D magnetic compounds.

It was most remarkable, therefore, when Kitanine, Maillet, and Terras [3] succeeded in reducing matrix elements between Bethe wave functions for local spin operators to determinantal expressions. Here we use these expressions and the norms previously determined by Korepin [4] to calculate dynamic spin structure factors of the Heisenberg antiferromagnet with periodic boundary conditions and a magnetic field:

$$
H=\sum_{n=1}^{N}\left[J \mathbf{S}_{n} \cdot \mathbf{S}_{n+1}-h S_{n}^{z}\right] .
$$

\section{Transition rates expressed as determinants}

Consider Bethe wave functions with $z$-component $S_{T}^{z}=$ $N / 2-r$ of the total spin. They are specified by sets of ra- pidities $z_{1}, \ldots, z_{r}$, which are solutions of the Bethe ansatz equations

$$
N \phi\left(z_{i}\right)=2 \pi I_{i}+\sum_{j \neq i} \phi\left[\left(z_{i}-z_{j}\right) / 2\right], \quad i=1, \ldots, r,
$$

where $\phi(z) \equiv 2 \arctan z$. The Bethe quantum numbers $I_{i}$ provide a natural classification of the spectrum. The challenge is to calculate transition rates for physically motivated operators from the information encoded in the $z_{i}$. Using the Bethe wave function directly is feasible but computationally inefficient and therefore limited to relatively small systems $(r<12)$ [5]. The combined advances reported in Refs. [3, 4] are the basis of a much more powerful approach, which will be used in the following to calculate transition rates

$$
M_{\lambda}^{\mu}(q) \equiv \frac{\left|\left\langle\psi_{0}\left|S_{q}^{\mu}\right| \psi_{\lambda}\right\rangle\right|^{2}}{\left\|\psi_{0}\right\|^{2}\left\|\psi_{\lambda}\right\|^{2}}, \quad \mu=z,+,-
$$

from the ground state of (11) for the operators

$$
S_{q}^{\mu}=\frac{1}{\sqrt{N}} \sum_{n} e^{i q n} S_{n}^{\mu}, \quad \mu=z,+,-.
$$

They probe the parallel $(\mu=z)$ and the perpendicular $(\mu=+,-)$ spin fluctuations at zero temperature.

The transition rates for the parallel spin fluctuations as inferred from Eq. (5.12) of Ref. [3] for the matrix element $\left\langle\psi_{0}\left|S_{n}^{z}\right| \psi_{\lambda}\right\rangle$ can be brought into the form

$$
M_{\lambda}^{z}(q)=\frac{N}{4} \frac{\left|\Omega^{z}\right|^{2}}{\left\|\psi_{0}\right\|^{2}\left\|\psi_{\lambda}\right\|^{2}},
$$


where

$$
\begin{gathered}
\Omega^{z}\left(\left\{z_{j}^{0}\right\}_{r},\left\{z_{j}\right\}_{r}\right)=\prod_{j=1}^{r} \frac{z_{j}^{0}+i}{z_{j}+i} \\
\times \frac{2^{r} \operatorname{det}(\overline{\mathrm{H}}-2 \overline{\mathrm{P}})}{\prod_{i<j}^{r}\left(z_{i}^{0}-z_{j}^{0}\right) \prod_{i>j}^{r}\left(z_{i}-z_{j}\right)}, \\
\overline{\mathrm{H}}_{a b}=\frac{i}{z_{a}^{0}-z_{b}} \\
\times\left(\prod_{j \neq a}^{r}\left(z_{j}^{0}-z_{b}+2 i\right)-d\left(z_{b}\right) \prod_{j \neq a}^{r}\left(z_{j}^{0}-z_{b}-2 i\right)\right) \\
\overline{\mathrm{P}}_{a b}=\frac{i}{\left(z_{a}^{0}\right)^{2}+1} \prod_{j=1}^{r}\left(z_{j}-z_{b}+2 i\right) \\
d\left(z_{i}\right)=\left(\frac{z_{i}-i}{z_{i}+i}\right)^{N} .
\end{gathered}
$$

The rapidities $\left\{z_{i}^{0}\right\}$ and $\left\{z_{i}\right\}$ belong to the ground state $\left|\psi_{0}\right\rangle$ and to the excited state $\left|\psi_{\lambda}\right\rangle$, respectively. The norm of $\left|\psi_{\lambda}\right\rangle$ from Ref. [4, transcribed to our notation, reads:

$$
\begin{gathered}
\left\|\psi_{\lambda}\right\|^{2}=\left(\prod_{i \neq j}^{r} \frac{z_{i}-z_{j}-2 i}{z_{i}-z_{j}}\right) \operatorname{det} \mathrm{N}\left(\left\{z_{i}\right\}\right), \\
\mathrm{N}_{a b}=-2 i \frac{\partial}{\partial z_{b}} \ln \left\{\left(\frac{z_{a}+i}{z_{a}-i}\right)^{N} \prod_{k \neq a}^{r} \frac{z_{a}-z_{k}-2 i}{z_{a}-z_{k}+2 i}\right\},
\end{gathered}
$$

and can be compactified into

$$
\left\|\psi_{\lambda}\right\|^{2}=\frac{2^{r^{2}} \operatorname{det} \mathrm{K}\left(\left\{z_{i}\right\}\right)}{\prod_{i<j}^{r} K\left(z_{i}-z_{j}\right) \prod_{i<j}^{r}\left(z_{i}-z_{j}\right)^{2}}
$$

where

$$
\begin{gathered}
\mathrm{K}_{a b}= \begin{cases}K\left(z_{a}-z_{b}\right) & : a \neq b \\
N \kappa\left(z_{a}\right)-\sum_{j \neq a}^{r} K\left(z_{a}-z_{j}\right) & : a=b\end{cases} \\
\kappa(z) \doteq \frac{2}{1+z^{2}}, \quad K(z) \doteq \frac{4}{4+z^{2}}
\end{gathered}
$$

For real $z_{i}$ the absolute square of (6) becomes:

$$
\left|\Omega^{z}\right|^{2}=\frac{2^{2 r} \prod_{i=1}^{r}\left[\kappa\left(z_{i}\right) / \kappa\left(z_{i}^{0}\right)\right]}{\prod_{i<j}^{r}\left(z_{i}^{0}-z_{j}^{0}\right)^{2} \prod_{i<j}^{r}\left(z_{i}-z_{j}\right)^{2}}|\operatorname{det}(\overline{\mathrm{H}}-2 \overline{\mathrm{P}})|^{2}
$$

Rescaling $\overline{\mathrm{H}}, \overline{\mathrm{P}}$ consolidates powers of two. The calculation of the transition rate is thus reduced to the evaluation of three $r \times r$ determinants:

$$
\begin{gathered}
M_{\lambda}^{z}(q)=\frac{N}{4} \frac{\mathcal{L}_{r}\left(\left\{z_{i}\right\}\right)}{\mathcal{L}_{r}\left(\left\{z_{i}^{0}\right\}\right)} \mathcal{K}_{r}\left(\left\{z_{i}^{0}\right\}\right) \mathcal{K}_{r}\left(\left\{z_{i}\right\}\right) \\
\times \frac{|\operatorname{det}(\mathrm{H}-\mathrm{P})|^{2}}{\operatorname{det} \mathrm{K}\left(\left\{z_{i}\right\}\right) \operatorname{det} \mathrm{K}\left(\left\{z_{i}^{0}\right\}\right)}, \\
\mathcal{L}_{r}\left(\left\{z_{i}\right\}\right) \doteq \prod_{i=1}^{r} \kappa\left(z_{i}\right), \quad \mathcal{K}_{r}\left(\left\{z_{i}\right\}\right) \doteq \prod_{i<j}^{r} K\left(z_{i}-z_{j}\right), \\
\mathrm{H}_{a b} \doteq \frac{i}{z_{a}^{0}-z_{b}} \\
\times\left(\prod_{j \neq a}^{r} G\left(z_{j}^{0}-z_{b}\right)-d\left(z_{b}\right) \prod_{j \neq a}^{r} G^{*}\left(z_{j}^{0}-z_{b}\right)\right) \\
\mathrm{P}_{a b} \doteq i 2 \kappa\left(z_{a}^{0}\right) \prod_{j=1}^{r} G\left(z_{j}-z_{b}\right), \\
\quad G(z) \doteq \frac{z}{2}+i .
\end{gathered}
$$

Along similar lines of manipulation, the transition rates

$$
M_{\lambda}^{ \pm}(q)=N \frac{\left|\Omega^{ \pm}\right|^{2}}{\left\|\psi_{0}\right\|^{2}\left\|\psi_{\lambda}\right\|^{2}},
$$

for the perpendicular spin fluctuations as inferred from the Eqs. (5.3) and (5.7) of Ref. [3] for the matrix element $\left\langle\psi_{0}\left|S_{n}^{+}\right| \psi_{\lambda}\right\rangle$ are brought into the form:

$$
\begin{aligned}
& M_{\lambda}^{ \pm}(q)=\left(\frac{\mathcal{L}_{r}\left(\left\{z_{i}^{0}\right\}\right)}{\mathcal{L}_{r \pm 1}\left(\left\{z_{i}\right\}\right)}\right)^{ \pm 1} \mathcal{K}_{r}\left(\left\{z_{i}^{0}\right\}\right) \mathcal{K}_{r \pm 1}\left(\left\{z_{i}\right\}\right) \\
& \times \frac{N\left|\operatorname{det} \mathrm{H}^{ \pm}\right|^{2}}{\operatorname{det} \mathrm{K}\left(\left\{z_{i}\right\}\right) \operatorname{det} \mathrm{K}\left(\left\{z_{i}^{0}\right\}\right)}, \\
& \mathrm{H}_{a b}^{+}=\frac{i}{z_{a}-z_{b}^{0}} \\
& \times\left(\prod_{j \neq a}^{r+1} G\left(z_{j}-z_{b}^{0}\right)-d\left(z_{b}^{0}\right) \prod_{j \neq a}^{r+1} G^{*}\left(z_{j}-z_{b}^{0}\right)\right) \\
& \mathrm{H}_{a, r+1}^{+}=i \kappa\left(z_{a}\right), \quad a=1, \ldots r+1, b=1, \ldots, r \\
& \mathrm{H}_{a b}^{-}=\frac{i}{z_{a}^{0}-z_{b}} \\
& \times\left(\prod_{j \neq a}^{r} G\left(z_{j}^{0}-z_{b}\right)-d\left(z_{b}\right) \prod_{j \neq a}^{r} G^{*}\left(z_{j}^{0}-z_{b}\right)\right) \\
& \mathrm{H}_{a r}^{-}=i \kappa\left(z_{a}^{0}\right), \quad a=1, \ldots r, b=1, \ldots, r .
\end{aligned}
$$




\section{Applications}

To demonstrate the efficacy of expressions (16) and (22) for the transition rates $M_{\lambda}^{\mu}(q), \mu=z,+,-$, we present three applications to the dynamic structure factors

$$
S_{\mu \bar{\mu}}(q, \omega)=2 \pi \sum_{\lambda} M_{\lambda}^{\mu}(q) \delta\left(\omega-\omega_{\lambda}\right)
$$

We begin with the parallel spin fluctuations at magnetization $M_{z} / N=\frac{1}{4}$ (half the saturation value). The spectral weight of $S_{z z}(q, \omega)$ was shown to be dominated by a set of collective excitations consisting of two unbound quasiparticles named $\psi$ and $\psi^{*}$ [6]. The spectral range of the $\psi \psi^{*}$ continuum, shown in Fig. 1(a), has soft modes at $q=0, \pi / 2$ and is partly folded back onto itself along a stretch of its upper boundary.

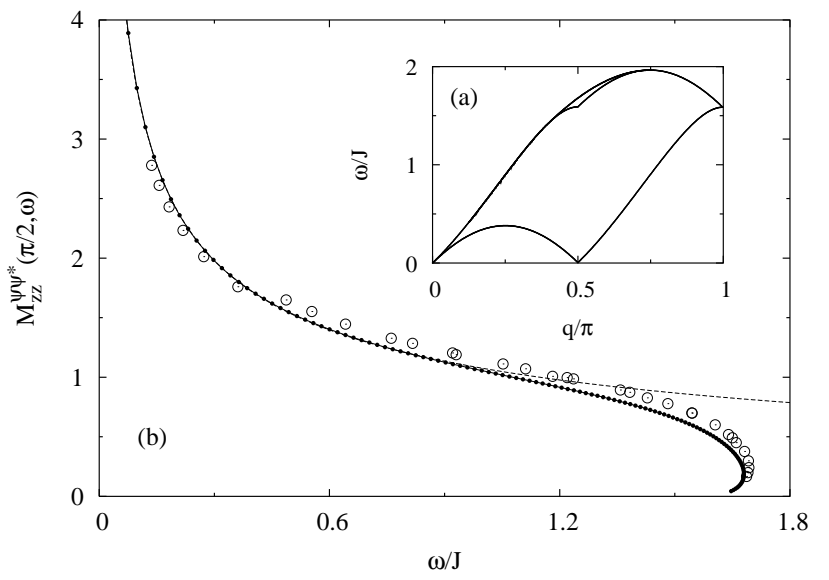

Fig. 1. (a) Energy versus wave number of the $\psi \psi^{*}$ excitations for $N \rightarrow \infty$. (b) Scaled transition rates between the ground state and the $\psi \psi^{*}$ states at $q=\pi / 2$ for $N=12,16, \ldots, 32$ (open circles) and $N=512$ (closed circles). The dashed line is a fit $a+b \omega^{\eta-2}$ of the $N=512$ data at $\omega / J \leq 0.5$. All results are for $M_{z}=N / 4$.

In Fig. 1 (b) we have plotted the scaled transition rates $M_{z z}^{\psi \psi^{*}}(\pi / 2, \omega)=N M_{\lambda}^{z}(\pi / 2)$ versus $\omega$ pertaining to the $\psi \psi^{*}$ excitations. The open circles are results previously obtained for $N \leq 32$ by calculating matrix elements directly from Bethe wave functions [5]. The full circles are data for $N=512$ as obtained from the determinantal expression (16).

The function $M_{z z}^{\psi \psi^{*}}(\pi / 2, \omega)$ varies smoothly across the entire $\psi \psi^{*}$ continuum including the fold. The observed infrared singularity, $M_{z z}^{\psi \psi^{*}}(\pi / 2, \omega) \sim \omega^{\eta-2}, \eta-2=-0.468 \ldots$ confirms exact predictions [7], 8]. At the other end of the continuum, $M_{z z}^{\psi \psi^{*}}(\pi / 2, \omega)$ tends to go to zero or a value close to zero.

The density $D^{\psi \psi^{*}}(\pi / 2, \omega)=2 \pi /\left[N\left(\omega_{i+1}-\omega_{i}\right)\right]$ of $\psi \psi^{*}$ states is shown in Fig. 2(a). It is flat and featureless except near the upper band edge, where the fold in the continuum produces a square-root divergence [6]. The spectralweight distribution $S_{z z}^{\psi \psi^{*}}(\pi / 2, \omega)$ resulting from the product of $M_{z z}^{\psi \psi^{*}}$ and $D^{\psi \psi^{*}}$ is then a double-peak structure as

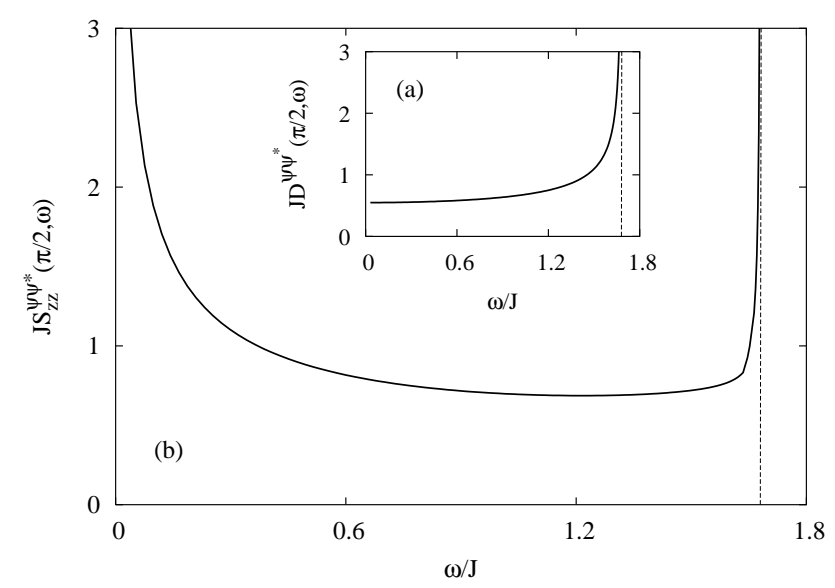

Fig. 2. (a) Density of $\psi \psi^{*}$ states and (b) spectral weight distribution of the $\psi \psi^{*}$ states in $S_{z z}(q, \omega)$ at $q=\pi / 2$ and $M_{z}=N / 4$ from data for $N=512$.

shown in Fig. 2(b) with the divergences at the lower and upper band edges caused by the transition rates and the density of states, respectively. At frequencies where the continuum is folded back, the spectral weight of two lines of excitations must be added up to produce the correct lineshape, causing a (barely visible) singularity within the band.

Now consider the perpendicular spin fluctuations as described by $S_{-+}(q, \omega)$, again at $M_{z} / N=\frac{1}{4}$. At the zone boundary $(q=\pi)$, some $98 \%$ of the spectral weight is carried by a continuum of collective excitations consisting of two $\psi$ quasiparticles [5]. The shape of the $\psi \psi$ continuum is shown in Fig. 3(a) The scaled transition rates $M_{-+}^{\psi \psi}(\pi, \omega)=N M_{\lambda}^{-}(\pi)$ pertaining to the $\psi \psi$ excitations are plotted versus $\omega$ in Fig. 3(b) for $N=1536$ by numerical evaluation of the determinantal expression (22). Also shown are data points for $N=12,16, \ldots, 28$ previously obtained by a different method [5].

Again we find a smooth variation of the transition rates across the continuum with distinct endpoint singularities. The infrared divergence is strong, $S_{-+}^{\psi \psi}(\pi, \omega) \sim$ $\omega^{1 / \eta-2}, \quad 1 / \eta-2=-1.346 \ldots$ as predicted $[7,8]$. At the upper band edge, the transition rates approach zero in what looks like a linear trend. The density $D^{\psi \psi}(\pi, \omega)$ of $\psi \psi$ states is again flat up to near the upper band edge, where it has a square-root divergence [Fig. 3(c)]. Unlike in the case of the $\psi \psi^{*}$ excitations discussed previously, here the divergence of the density of states coincides with a zero in the transition rates. In the resulting lineshape, shown in Fig. 3(d), the divergence is thus suppressed and converted into a cusp.

In the limit of zero external magnetic field $(h \rightarrow 0)$, the $\psi \psi$ continuum turns into the more familiar two-spinon continuum. The exact two-spinon lineshape of $S_{-+}(\pi, \omega)$ as previously obtained for $N=\infty$ via algebraic analysis [9] does indeed exhibit features very similar to those of $S_{-+}^{\psi \psi}(\pi, \omega)$ observed here for the first time. In the zerofield case, however, the power-law singularities are accompanied by logarithmic corrections. 

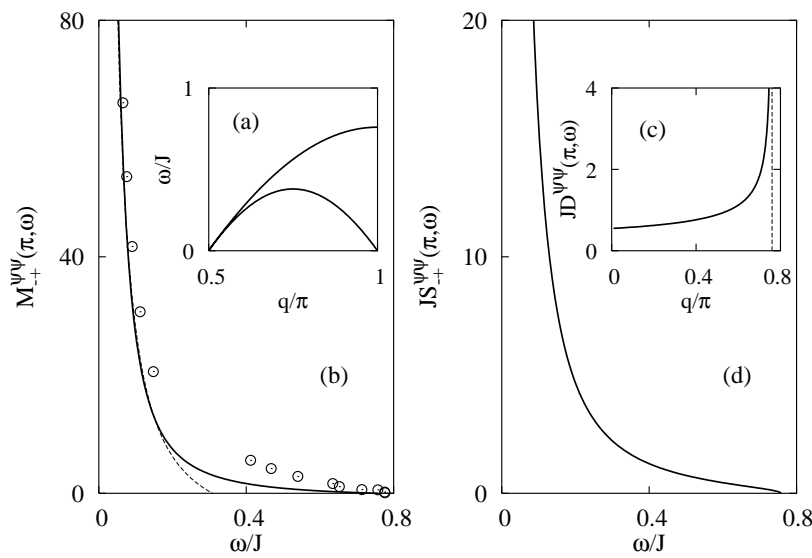

Fig. 3. (a) Continuum of $\psi \psi$ excitations. (b) Scaled transition rates between the ground state and the $\psi \psi$ states at $q=\pi$ for $N=12,16, \ldots, 28$ (open circles) and $N=1536$ (solid line). The dashed line is a fit $a+b \omega^{1 / \eta-2}$ of the $N=1536$ data at $\omega \leq 0.25$. (c) Density of $\psi \psi$ states at $q=\pi$ for $N=1536$. (d) Lineshape at $q=\pi$ of the $\psi \psi$ contribution to $S_{-+}(q, \omega)$. All results are for $M_{z}=N / 4$.

Our concluding application pertains to the perpendicular spin fluctuations as described by $S_{+-}(q, \omega)$, for which we again use the transition rates (22). Whereas the function $S_{-+}(q, \omega)$ dominates the perpendicular spin fluctuations in weak magnetic fields, it is the function $S_{+-}(q, \omega)$ that carries most of the spectral weight in strong fields.

A set of dynamically dominant collective excitations is identified which consists of unbound pairs of $\psi$ and $\psi^{*}$ quasiparticles as already encountered in the parallel spin fluctuations. However, since the perpendicular and parallel spin fluctuation operators reach these excitations in different invariant Hilbert subspaces, the spectral boundaries of the $\psi \psi^{*}$ spectrum in $S_{+-}(q, \omega)$ are related to those shown in Fig. 1(a) by reflection at the line $q=\pi / 2$ [10,5].

One outstanding feature of the spectral-weight distribution $S_{+-}^{\psi \psi}(q, \omega)$ is the distinct scaling behavior of the transition rates for the $\psi^{*}$ branch of the lower boundary [5]. Figure 4(a) shows the energy-momentum relation of this set of excitations for various values of $M_{z} / N$. This particular branch only exists for $M_{z} \neq 0$. With $M_{z}$ increasing from zero, it emerges at $\omega=0, q=0$. The frequency of its member state at $q=0$ increases proportional to $h$ and the wave number of its member state at $\omega=0$ increases proportional to $M_{z}$. Upon saturation $\left(M_{z} / N \rightarrow 1 / 2\right)$, it turns into the branch of one-magnon states with dispersion $\omega(q)=J(1+\cos q)$. In Fig. Al(b) we plot the unscaled transition rates $M_{\lambda}^{+}(q)$ via (22) of these states versus $q$ for the same values of $M_{z} / N$ as in panel (a). Also shown are two additional values very close to saturation. The $N$-dependence at fixed $M_{z} / N$ of these transition rates is very weak, in particular at small $q$. We have seen that excitations belonging to a continuum have transition rates with very different scaling behavior.

The $\psi^{*}$ transition rate at $q=0$ is exactly known for arbitrary values of $M_{z}: S_{+-}(0, \omega)=\left(2 M_{z} / N\right) 2 \pi \delta(\omega-h)$. It represents a resonant mode of the field-induced magne-
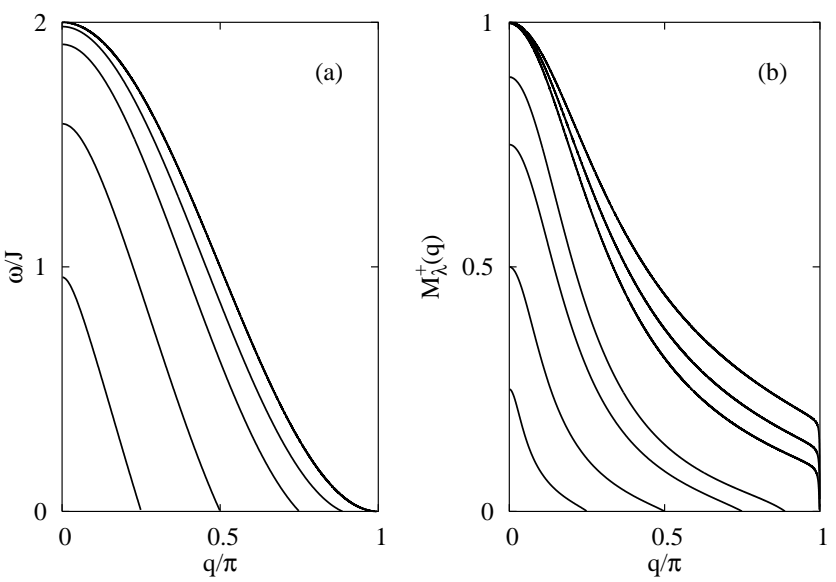

Fig. 4. (a) Energy-momentum relation at $M_{z} / N=192 / 1536=$ $0.125, \quad 384 / 1536=0.25, \quad 576 / 1536=0.375, \quad 1364 / 3072 \simeq$ $0.444,9980 / 20000=0.499$ of the $\psi^{*}$ branch that is part of the lower boundary of the $\psi \psi^{*}$ continuum. (b) Unscaled transition rates for the states shown in (a) and those at $M_{z} / N=$ $9990 / 20000=0.4995,9995 / 20000=0.49975$.

tization. In the limit $h \rightarrow h_{S}=2 J$, the transition rates become independent of $q$ and carry $100 \%$ of the intensity, $S_{+-}(q, \omega)=2 \pi \delta(\omega-2 J)$, which is well understood in the context of magnon excitations [10]. This trend is very slow but clearly visible in the finite- $N$ transition rate data of Fig. 目(b).

What is perhaps most surprising is that any one of these modes carries a nonzero fraction of the spectral weight already far below saturation. Hence they manifest themselves in sharp resonance lines, separate from the adjacent continuous spectral weight distributions, with intensities that become stronger as the magnetic field increases.

Financial support from the DFG Schwerpunkt "Kollektive Quantenzustände in elektronischen 1D Übergangsmetallverbindungen" (for M.K.) is gratefully acknowledged.

\section{References}

1. H. Bethe Z. Phys., 71:205, 1931.

2. V. E. Korepin, N. M. Bogoliubov, and A. G. Izergin. Quantum Inverse Scattering and Correlation Functions, Cambridge University Press, 1993.

3. N. Kitanine, J. M. Maillet, and V. Terras. Nucl. Phys. B, 554:647, 1999.

4. V. E. Korepin. Commun. Math. Phys., 86:391, 1982.

5. M. Karbach, D. Biegel, and G. Müller. Phys. Rev. B, (submitted).

6. M. Karbach and G. Müller. Phys. Rev. B, 62:14871, 2000.

7. F. D. M. Haldane. Phys. Rev. Lett., 45:1358, 1980.

8. A. Fledderjohann, C. Gerhardt, K.-H. Mütter, A. Schmitt, and M. Karbach. Phys. Rev. B, 54:7168, 1996.

9. M. Karbach, G. Müller, A. H. Bougourzi, A. Fledderjohann, and K.-H. Mütter. Phys. Rev. B, 55:12510, 1997. 
10. G. Müller, H. Thomas, H. Beck, and J. C. Bonner. Phys. Rev. B, 24:1429, 1981. 